\title{
A G.984 GPON Exhibiting Multi-Wavelength Protocol Functionalities
}

\author{
Ali Gliwan, Pandelis Kourtessis, and John M Senior \\ Optical Networks Group, \\ Science and Technology Research Institute (STRI) \\ University of Hertfordshire, Hatfield, UK \\ \{A.Gliwan, P.Kourtessis, J.M.Senior\}@Herts.ac.uk
}

\begin{abstract}
A Gigabit Passive Optical Network (GPON) upstream-map frameformat enhancement has been developed to accommodate dynamic multi wavelength (DMW) transmission over splitter-based GPONs. Accordingly network functionality has been supported by means of an algorithm, managing bandwidth allocation among utilised wavelengths in a dynamic two dimensional protocol. Modelling of the performance characteristics of the DMW-GPON topology in OPNET has demonstrated a minimum of $100 \mathrm{Mbit} / \mathrm{s}$ bandwidth provision for each of 32 Optical Network Units (ONUs) with a maximum $0.085 \mathrm{~s}$ packet delay for the worst performing lower service level agreement (SLA) ONUs.
\end{abstract}

Keywords: DBA; DWA; WDM; GPON; DMW.

\section{Introduction}

Scalability of standard GPON [1] topologies to larger split ratios would result in increased upstream polling waiting-time that could be intolerable for real-time services. The application of wavelength division multiplexing (WDM) to assign each ONU a unique point-to-point (P2P) logical connection with the optical line termination (OLT) provides a solution for reducing the polling waiting time and relaxing the bandwidth requirements on optical and electrical components. At present coarse WDM operation over the currently deployed splitter-based PONs has been proposed by means of the GPON band enhancement, defined by the ITU-T [2].

The application of extended band overlay has been demonstrated over a standard GPON topology and provided an ideal interim solution for smooth, dynamic and ondemand capacity upgrade [3]. It was achieved in [3] by reviewing the upstream and downstream frame format maps and consequently developing a new protocol based on the dynamic bandwidth allocation (DBA) algorithms previously developed for singlewavelength GPONs. To adapt and extend these algorithms for coarse WDM operation, extra fields were incorporated into the GPON frame format \{ITU-T, $2004 \# 74$ \}. Both the grant and the report packets used to establish communication between the OLT and ONUs were reconfigured to support dynamic multi-wavelength operation. Out of the twelve bits, in the Flags-field of the GPON upstream map frame format, the six unused 
reserved bits were utilised by assigning four bits to express the ONU's operating wavelength for proceeding cycles and two bits to specify the packet type (e.g. whether it is a control packet or data packet as shown in Fig. 1).

\begin{tabular}{|c|c|c|c|c|c|}
\hline \multirow{2}{*}{$\begin{array}{c}\text { Alloc_ID } \\
12 \text { Bits }\end{array}$} & \multicolumn{2}{|c|}{ Flags 12 Bits } & \multirow{2}{*}{$\begin{array}{l}\text { SStart } \\
2 \text { Bytes }\end{array}$} & \multirow{2}{*}{$\begin{array}{l}\text { SStop } \\
2 \text { Bytes }\end{array}$} & \multirow{2}{*}{$\begin{array}{l}\text { CRC } \\
8 \text { Bits }\end{array}$} \\
\hline & Reserved 6 Bits & Used 6 Bits & & & \\
\hline \multirow{2}{*}{$\begin{array}{l}\text { Alloc_ID } \\
12 \text { Bits }\end{array}$} & \multicolumn{2}{|c|}{ Flags 12 Bits } & \multirow{2}{*}{$\begin{array}{l}\text { SStart } \\
2 \text { Bytes }\end{array}$} & \multirow{2}{*}{$\begin{array}{l}\text { SStop } \\
2 \text { Bytes }\end{array}$} & \multirow{2}{*}{$\begin{array}{l}\text { CRC } \\
8 \text { Bits }\end{array}$} \\
\hline & $\begin{array}{c}\text { Op_Wavln } \\
4 \text { Bits }\end{array} \underbrace{}_{\begin{array}{c}\text { Type } \\
2 \text { Bits }\end{array}}$ & Used 6 Bits & & & \\
\hline
\end{tabular}

Fig. 1. Upstream map frame format, (a) Single-wavelength GPON, (b) DMW- GPON.

The wavelengths utilised by each ONU for data transfer could be defined during the ONU's registration stage by means of each ONU reporting its supported wavelengths to the OLT [3]. To demonstrate the maximum transmission time-slot utilisation for each operating wavelength and consequently provide reduced packet delay, a scheduling algorithm was developed that prioritises user transmission according to traffic status.

\section{The Dynamic Multi-Wavelength Protocol}

The DMW protocol aims to increase the upstream bit-rate by introducing dynamic allocation of bandwidth concurrently in the wavelength and time domains. This has been initially achieved by developing the dynamic minimum bandwidth (DMB) algorithm [4, 5] and modifying the GPON frame format to support multi-wavelength operation. The DMB algorithm facilitates three SLAs to assign each ONU with a guaranteed minimum bandwidth, to satisfy their basic service requirements, plus an additional allocation of extra bandwidth on demand based on the assigned SLA. In the DMW algorithm presented here a fourth service level is introduced to comply with modern service level provisioning [6] and to provide greater user experience and network flexibility. In addition, an extra upstream wavelength, bringing the total to five, is introduced with respect to previous DMW developments [3] to scale-up wavelength assignment in view of the ITU-T G.984.5 standard [2].

During ONU registration, the OLT "requests" from each ONU to confirm their supported wavelength(s). This is crucial to distinguish between different network sectors and bandwidth provision status among ONUs. Consequently, the OLT assigns the upstream bandwidth available in each polling-cycle in three stages. In the first stage, and after having received the requested bandwidth from each ONU, the OLT calculates a safety margin as shown in Fig. 2. The purpose of this approach is to process the maximum cycle time for bandwidth allocation independently for each wavelength, allowing for more accurate population of the polling cycles with considerable decrease in idle time slots. The safety margin is determined by considering the overall ONU minimum bandwidth requirement and the ONU SLA contracts. In that manner, the OLT allocates ONU bandwidth by means of the individual total network capacity depending on the time and wavelength measures ( $R_{\text {Available }}$ ), as seen in equation (1). 


$$
\mathbf{R}_{\text {Avaiable }}=\sum_{\lambda=0}^{\mathrm{n}} \mathbf{R}_{\text {Max }}^{\lambda}-\text { Safety margin }
$$

The maximum allowed bandwidth for $\mathrm{ONU}_{\mathrm{i}}\left(B_{\text {allowed }}^{t}\right)$ is then assigned according to the DMB algorithm $[4,5]$ as in equation (2).

$$
B_{\text {allowed }}^{t}=\left\{\begin{array}{ccc}
B_{\text {req }}^{\text {ONUi }} & \text { if } & B_{\text {req }}^{\text {ONUs }}\left\langle R_{\text {Available }}\right. \\
B_{\max }^{t} & \text { if } & \left.B_{\text {req }}^{\text {ONUs }}\right\rangle R_{\text {Available }}
\end{array}\right.
$$

where:

$\mathrm{R}_{\text {Max }}^{\lambda} \quad$ is the maximum bit-rate for wavelength $\lambda$

$B_{r e q}^{O N U i}$ is the requested bandwidth by $\mathrm{ONU}_{\mathrm{i}}$

$\begin{array}{ll}B_{r e q}^{O N U s} & \text { is the total requested bandwidth by the network } \\ \mathrm{ONU}_{\mathrm{s}}\end{array}$

$B_{\max }^{t}$

is the maximum allowed bandwidth for SLA $(\mathrm{t})$

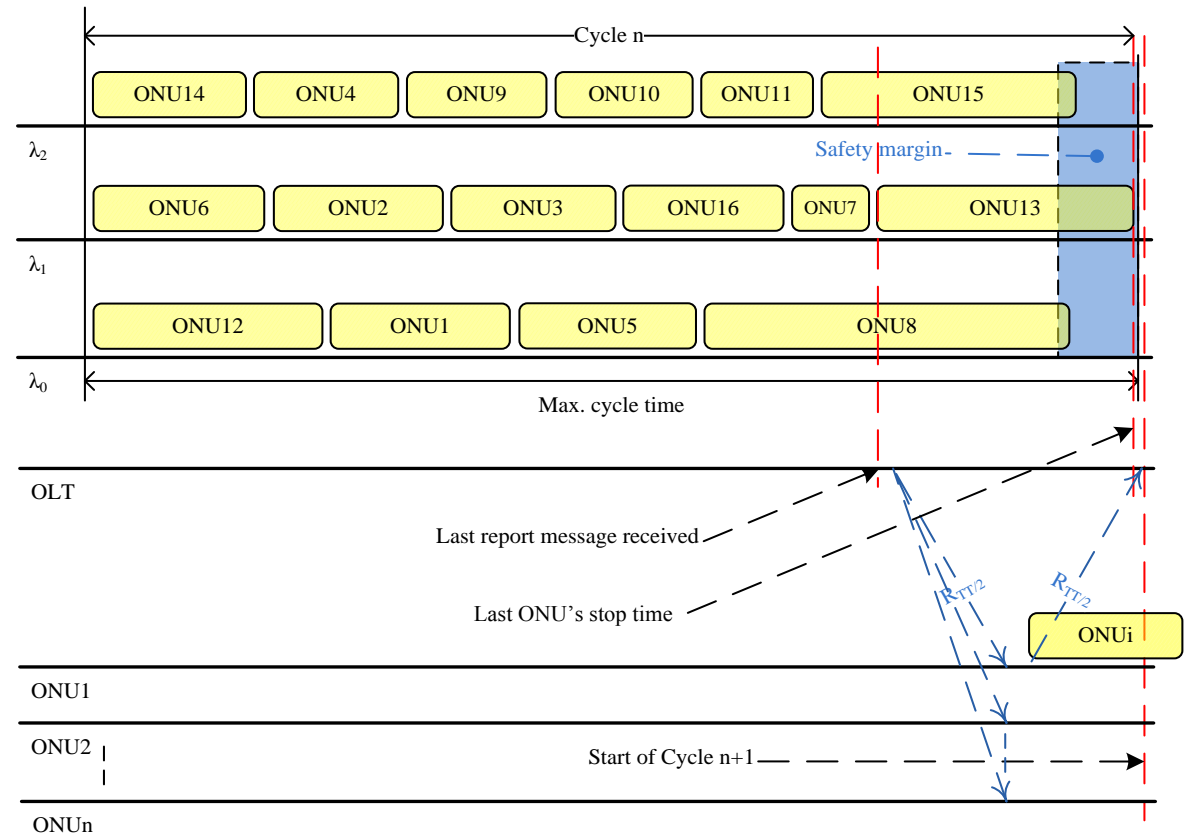

Fig. 2. Principles of the DMW-GPON bandwidth assignment.

Random distribution of ONU traffic among the different wavelengths could potentially result in exceeding the maximum available cycle-time. Progressively the 
DMW OLT specifies the three highest ONU allocated bandwidths, since three wavelengths are utilised in the current algorithm, and positions them at the end of each cycle as shown in Fig. 2.

As a result the network throughput is increased by decreasing adjacent cycle idle times, resulting from delays associated with processing the report and grant messages by the OLT and the associated round trip time (RTT). The remaining ONU bandwidths are assigned to wavelengths in order, from high to low, starting at $\lambda_{u p 0}$ and finishing at $\lambda_{\text {up } 2}$.

This process allows the OLT to guarantee that the last ONU time-slot in $\lambda_{\text {up } 2}$ can fit within the safety margin as shown in Fig. 2. This approach potentially produces a shorter polling cycle length, a reduction in the ONU upstream packet waiting-time in proceeding cycles, and hence increased network utilization. Although the DMW algorithm deals successfully with adjacent cycle idle times, the network wavelengths are not always evenly populated resulting in idle times within each cycle, as seen primarily in Fig. 2 for $\lambda_{u p 0}$ and $\lambda_{u p 2}$. To resolve this situation, before sending the gate message to the ONUs, the OLT will allocate any remaining available bandwidth for each wavelength to its assigned ONUs according to their SLA as seen in Fig.3.

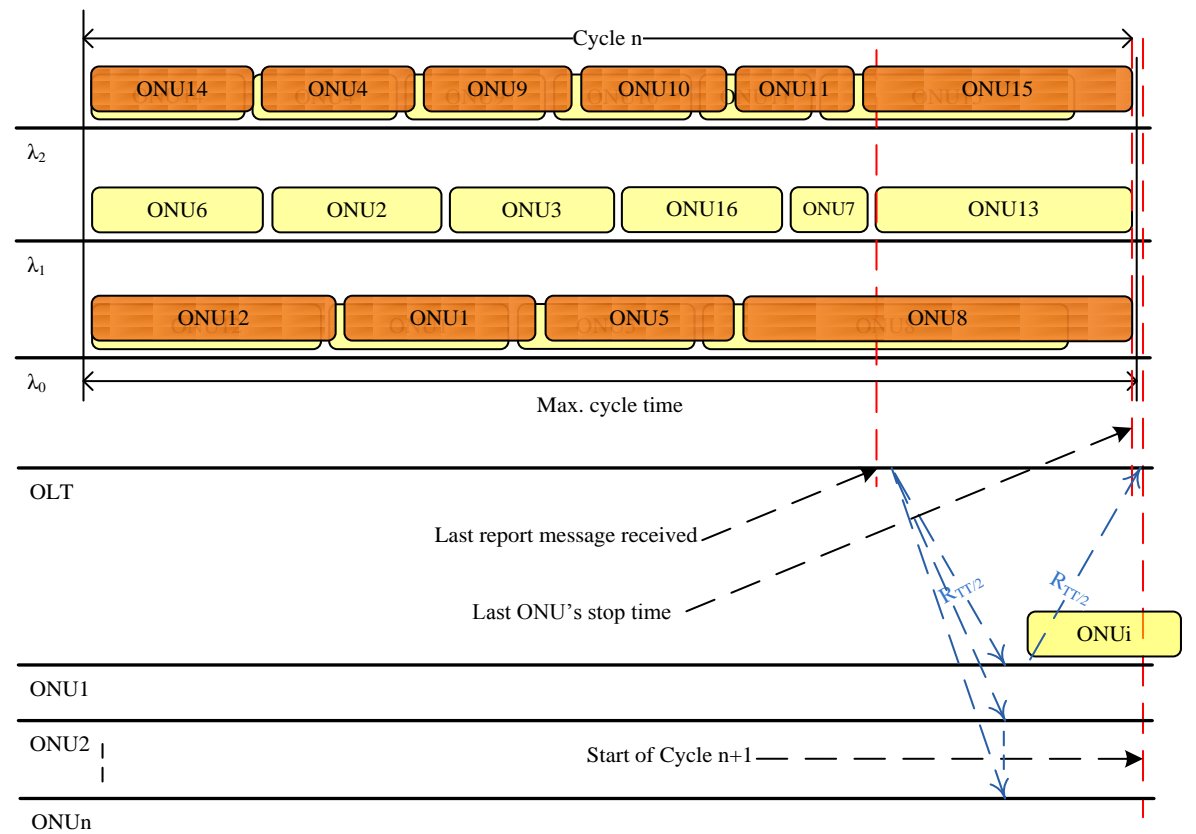

Fig. 3. DMW-GPON with extra bandwidth assignment.

Fig. 3 portrays the finalised bandwidth allocation for each ONU in orange on top of the initial allocation in yellow to demonstrate the protocol enhancement. This step leads to a better bandwidth utilisation and reduces the mean packet delay of the system. 


\section{Simulation Results}

To investigate the performance of the DMW protocol a FTTH oriented GPON network was modelled using the OPNET v.14.5 platform with Pareto self-similar traffic with typical Hurst parameter of 0.8, 1.24416 Gbit/s upstream data-rate, 2.488 Gbit/s downstream data-rate, and 32 ONUs. The latter were organised to implement service level agreement diversity with 4 ONUs assigned at $\mathrm{SLA}_{0}, 4 \mathrm{ONUs}_{\text {a }} \mathrm{SLA}_{1}, 8$ ONUs at $\mathrm{SLA}_{2}$, and 16 ONUs at SLA 3 from high SLA to low respectively, simulating progressive network usage. In particular a 96 bits GPON guard-time between ONU traffic was considered, a $2.0 \mathrm{~ms}$ maximum cycle time, $20 \mathrm{~km}$ link lengths between the OLT and ONUs, and as explained in the previous section, $3 \%$ of the maximum cycle time utilised as a safety margin.

Comparing the DMW-GPON performance, with that of the DMB-GPON, as shown in Fig. 4, the overall network capacity of the former is confirmed to have been increased by a factor equal to the number of wavelengths employed.

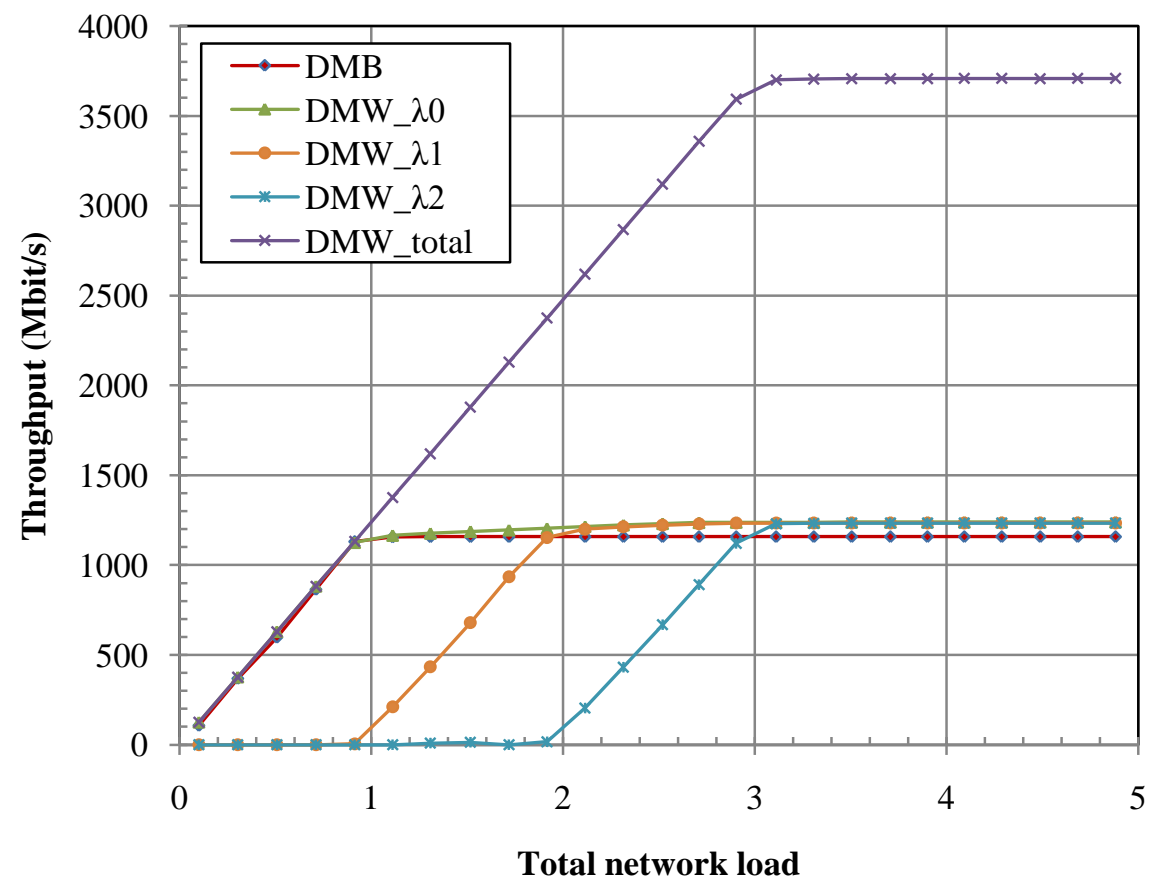

Fig. 4. Throughput against total network load for DMB and DMW.

Although such a characteristic was rather expected, it was achieved with the simultaneous increase in each DMW wavelength throughput by $70 \mathrm{Mbit} / \mathrm{s}$ compared to the DMB network. It has also been achieved at the provision of $100 \mathrm{Mbit} / \mathrm{s}$ minimum transmission rate per ONU, for $32 \mathrm{ONUs}$, as opposed to $30 \mathrm{Mbit} / \mathrm{s}$ basic bandwidth per ONU in the DMB-GPON. This imposes a reduction in idle time between cycles. In addition, the use of the $3 \%$ safety margin has not limited each wavelengths' 
throughput, in contrast to the single wavelength DMB capacity, due to the more effective utilisation of the available time slots.

In another performance evaluation measure, while the mean packet delay for all SLAs utilising a single wavelength exceeds $0.1 \mathrm{~s}$, when the total network load achieves one wavelength capacity at $1.24 \mathrm{Gbit} / \mathrm{s}$, the DMW protocol provides notably decreased packet delay. Corresponding figures indicate $0.003 \mathrm{~s}$ delay up to the point the total network load reaches three wavelengths capacity. Shown in Fig. 5, this characteristic allows for the non obstructive transmission of interactive applications since it satisfies the recommended one-way delay requirement for these applications as defined by the ITU-T G.1010 [7].

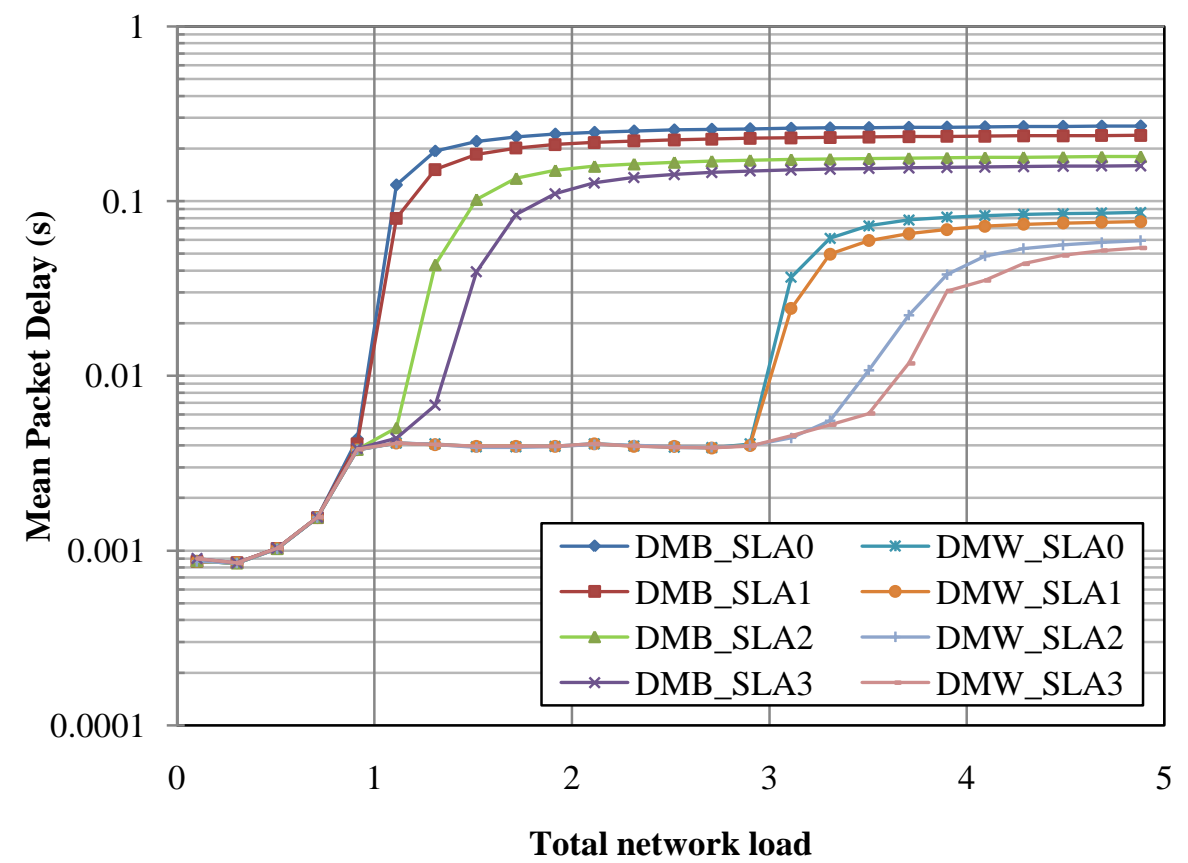

Fig. 5. Mean packet delay against total network load for DMB and DMW GPON.

The DMW protocol therefore allows for real time service provisioning at the stringiest requirements of service provisioning such as conversational voice and videophone, at increased basic bandwidth to $100 \mathrm{Mbit} / \mathrm{s}$ and increased ONU volume. Even at higher network load, the lower service level DMW ONUs displaying the greatest delay still maintain delay figures below $0.1 \mathrm{~s}$ which provides scope for further scalability to ONU provision and for service advancements. 


\section{Conclusions}

A DMW algorithm methodology and the corresponding protocol enhancements have been presented to accommodate multi-wavelength operation over splitter-based GPONs by means of smoothly upgrading the existing single-wavelength network infrastructure. This upgrade has been achieved by utilising additional bits in the frame fields of the GPON upstream format map to define the operating wavelength and packet type transfer for each ONU. The performance benefits of the multi-wavelength operation include aggregating transmission bit-rates of $3.70 \mathrm{Gbit} / \mathrm{s}$ in the presence of three wavelength signals and 32 ONUs, $100 \mathrm{Mbit} / \mathrm{s}$ minimum bandwidth per ONU and a considerable reduction in mean packet delay in comparison to a single wavelength GPON topology. The $0.003 \mathrm{~s}$ packet delay at typical network utilisation rates and the worst case $0.085 \mathrm{~s}$ equivalent at heavy loading allow for the continuous communication of the highest specification interactive services and a scope for additional network upgrade in services, ONU penetration and reach capabilities.

\section{References}

1. ITU-T.: G.984.2 Gigabit-capable Passive Optical Networks (G-PON): Physical media dependent (PMD) layer specification, ITU-T, (2003)

2. ITU-T.: G.984.5 Enhancement band for gigabit capable optical access networks, ITU-T, (2007)

3. Gliwan, A., Chang, C.H., Shachaf, Y., Kourtessis, P., Senior, J.M.: Upstream Format Map Enhancements for Multi-Wavelength GPONs. In: 13th European Conference on Networks and Optical Communications (NOC), pp. 75--82. Krems, Austria, (2008)

4. Chang, C.H., Kourtessis, P., Senior, J.M.: GPON service level agreement based dynamic bandwidth assignment protocol. IET Electronics Letters, pp. 1173--1174. (2006)

5. Chang, C.H., Alvarez, N. M., Kourtessis, P., Lorenzo, R. M.Senior, J.M.: Full-Service MAC Protocol for Metro-Reach GPONs. Journal of Lightwave Technology, pp. 1016--1022. (2010)

6. Super-Fast Cable Broadband, http://www.virginmedia.com

7. ITU-T.: G.1010 End-user multimedia QoS categories, ITU-T, (2001) 